\title{
NMR Polarization Measurements for Jefferson Lab's Solid Polarized Targets
}

\author{
James Maxwell*广 \\ Thomas Jefferson National Accelerator Facility \\ E-mail: jmaxwell@jlab.org
}

\begin{abstract}
Solid polarized targets rely on Nuclear Magnetic Resonance techniques to provide accurate measurements of the enhanced polarization provided by Dynamic Nuclear Polarization. Upcoming polarized target experiments in Jefferson Lab's Hall B present challenging conditions which demand improvements to traditional NMR methods. We will discuss the changes prompted by the new Hall B target, lay out plans for improvements to our NMR system, and show results of initial tests of our designs.
\end{abstract}

XVII International Workshop on Polarized Sources, Targets \& Polarimetry 16-20 October 2017

Kaist, South Korea

\footnotetext{
*Speaker.

${ }^{\dagger}$ On behalf of the Jefferson Lab Target Group and CLAS12 Polarized Target collaboration.
} 


\section{NMR Measurements for Dynamic Nuclear Polarization}

Dynamic nuclear polarization (DNP) is a mainstay of polarized scattering experiments such as those performed at Jefferson Lab [1], capable of providing in excess of $95 \%$ polarization of protons (in $\mathrm{NH}_{3}$ ) or $50 \%$ polarization of deuterons (in $\mathrm{ND}_{3}$ ). DNP takes advantage of the very high electron polarization $(P=\tanh (\mu B / k T)))$ at a practical temperature $(T \sim 1.4 \mathrm{~K})$ and magnetic field $(B \sim 5 T)$, to polarize nearby nuclei via flip-flop transitions in the electron-spin-nucleus-spin system. These transitions are driven by microwave radiation of frequency close to the electron spin resonance frequency less or more the nuclear Larmor frequency $\left(\omega_{\mathrm{esr}} \pm \omega_{\mathrm{nmr}}\right)$.

To measure the net polarization achieved in a sample, Nuclear Magnetic Resonance (NMR) methods are used. A coil of inductance $L_{0}$ is embedded in the sample, perpendicular to the polarizing field $B$, so that the created field $B_{0}$ at the Larmor frequency $\omega_{0}$ induces a spin flip, driving a transition of the Zeeman-splitting energy levels, $\hbar \omega_{0}=g \mu B$. Depending on the average orientation of the spins to the field, these spins flips absorb or emit energy as they interact with the field generated by the inductor and its electrical circuit.

The spin system's response to the induced RF field $B_{0}$ is its magnetic susceptibility, $\chi(\omega)$, and is a function of the RF frequency $\omega$. The magnetic susceptibility can be expressed as a difference of a dispersive term $\chi^{\prime}(\omega)$ and an absorptive term $\chi^{\prime \prime}(\omega)$. This absorptive term can be integrated in frequency to give a proportional measure of the target polarization $P$ :

$$
\chi(\omega)=\chi^{\prime}(\omega)-i \chi^{\prime \prime}(\omega), \quad P=K \int_{0}^{\infty} \chi^{\prime \prime}(\omega) d \omega
$$

The constant $K$ contains information on the nucleus in question as well as the properties of the NMR system. Since $\chi^{\prime \prime}(\omega)$ is non-zero only in a small range around the Larmor frequency, the integral only need be performed very close to center. The inductance of the embedded coil couples to the magnetic susceptibility, via a filling factor $\eta$,

$$
L_{C}(\omega)=L_{0}[1+4 \pi \eta \chi(\omega)]
$$

so that a measurement that isolates the real part of the complex impedance of the coil versus frequency will give a proportional measure of the polarization.

Liverpool Q-meters [2] have typically been used to perform such measurements for polarized targets applications including scattering experiments at Jefferson Lab. Traditionally, the Q-meter observes a tank (LCR) circuit with the resistor and capacitor within the Q-meter connected via a $\lambda / 2$ cable to the inductor embedded in the material at $1 \mathrm{~K}$, as shown in figure $2 \mathrm{a}$. The tank circuit's adjustable capacitor allows the tuning of the circuit so that the resonant frequency, $\omega_{0}=1 / \sqrt{L C}$, can be set to the Larmor frequency of the nucleus of interest. The Q-meter's key component is a balanced ring modulator, a type of frequency mixer which compares the signal from the tank circuit to the reference excitation RF frequency and returns the real portion of the signal (assuming the signal and reference match phase).

To perform a measurement, the reference signal is swept through frequency, starting below the Larmor frequency (213 MHz-400 kHz for $5 \mathrm{~T}$ protons) and increased in discrete steps. For a welltuned circuit, the Q-meter response results in a polynomial background curve versus frequency, known as the Q-curve, from reactive complex impedance below and above the resonant frequency. 
Any effect from the material polarization, such as the proton thermal equilibrium polarization signal seen in figure 1, is integrated by first removing this background Q-curve (or baseline). Thermal equilibrium (TE) measurements such as this one at known temperature and magnetic field allow the calibration of the signal area when the polarization is enhanced using DNP.

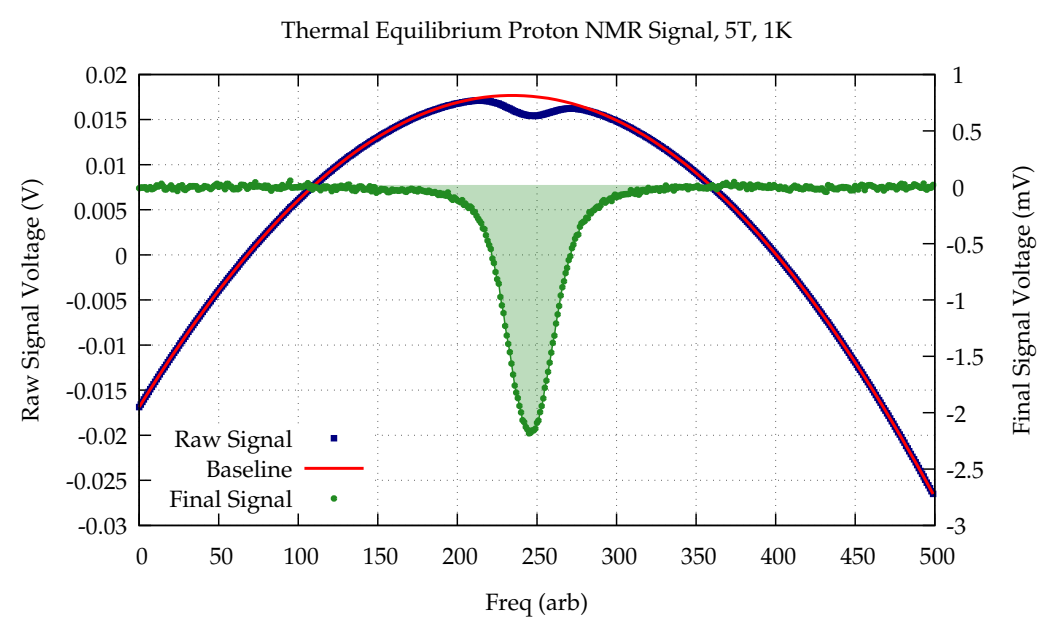

Figure 1: A Proton TE signal at $5 \mathrm{~T}$ and roughly $1 \mathrm{~K}$, showing the raw Q-meter response in blue, a polynomial fit to the baseline in red, and the resulting NMR signal after subtraction in green.

Traditionally, the tuning of the Q-meter NMR circuit has involved three basic steps. First, the length of the cable between the coil and the resistor and adjustable capacitor must be chosen to be an integer multiple of half the resonant wavelength $(n \lambda / 2)$ to remove the effect of the cable's impedance. Then, the tank circuit is tuned to $\omega_{0}$ by adjusting the capacitance in the Q-meter. Finally, the phase between the tank signal and the reference signal must be matched by adjusting the length of cable that the reference takes into the mixer, centering the real portion of the signal at $\omega_{0}$.

\subsection{NMR for the CLAS12 Polarized Target}

Building on the success of the polarized target program in Hall B during Jefferson Lab's $6 \mathrm{GeV}$ era, a new polarized target is being developed for the new CLAS12 detector package [3]. The new polarized target system will fit inside the 5 T CLAS12 solenoid, and is being designed to accommodate two target sample cells in the beam simultaneously. These cells, $2 \mathrm{~cm}$ long by $2 \mathrm{~cm}$ in diameter, will sit on the axis of the beam, in the center of the target cryostat, separated horizontally by roughly $3 \mathrm{~cm}$. Using 2 sets of shim coils to increase and decrease the magnetic field by approximately 80 gauss over the extent of either cell, single microwave frequency will be used to polarize each cell to opposite polarities.

While such a two-cell configuration has been successfully implemented at SMC [4] and more recently COMPASS [5], they both utilized a single magnetic field and two different microwave frequencies. The use of a single microwave frequency requires precise control over the shimmed fields and the frequency of the NMR measurement to operate smoothly. In the case of a traditional static polarizing field, the microwave frequency is chosen to maximize the polarization, and 
the optimal frequency changes as the material accumulates radiation damage [6,7]. With a static microwave frequency, the field of each cell will need to be changed independently as dose is accumulated. As the field changes, so does the NMR frequency. The NMR system must be able to be tuned remotely for such a scheme to work in an experimental setting, and this is not possible in the traditional system with rotary capacitors and cable length adjustment of the phase.

\section{Development}

A number of improvements to Jefferson Lab's solid polarized target NMR system are being implemented for use in the CLAS12 polarized target. First, we will revisit the "cold" circuit NMR technique which moves the resistor and tuning capacitor into the cryostat [8], as seen in figure $2 b$, removing the need for a $\lambda / 2$ cable between those components and the coil, reducing thermal noise. To this non-resonant-cable style circuit, we can also introduce varactor-diode variable capacitors to allowing remote tuning of the tank circuit [9]. To avoid the need to change the length of cable for phase matching we have included commercially available electronic phase-shifters. In addition to these incremental improvements, we are looking at ways to renovate or replace the aging Liverpool Q-meter systems.

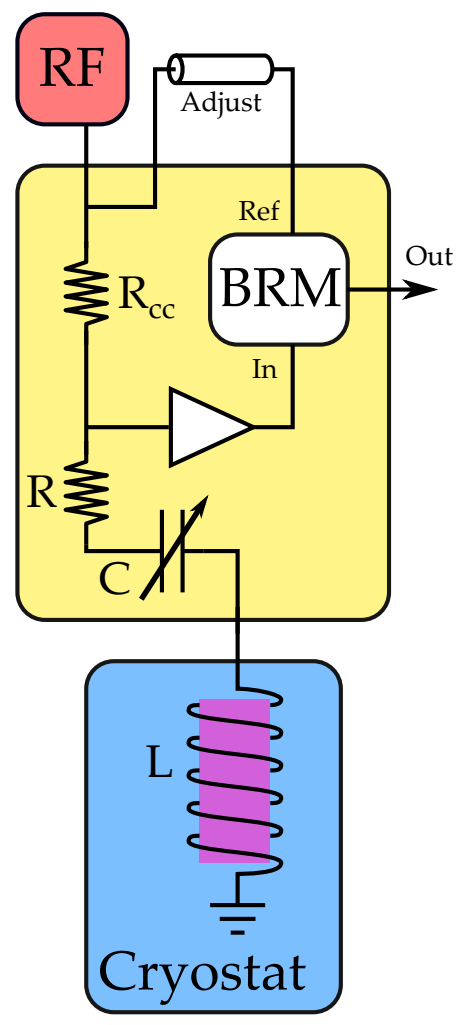

(a) Traditional Q-meter NMR

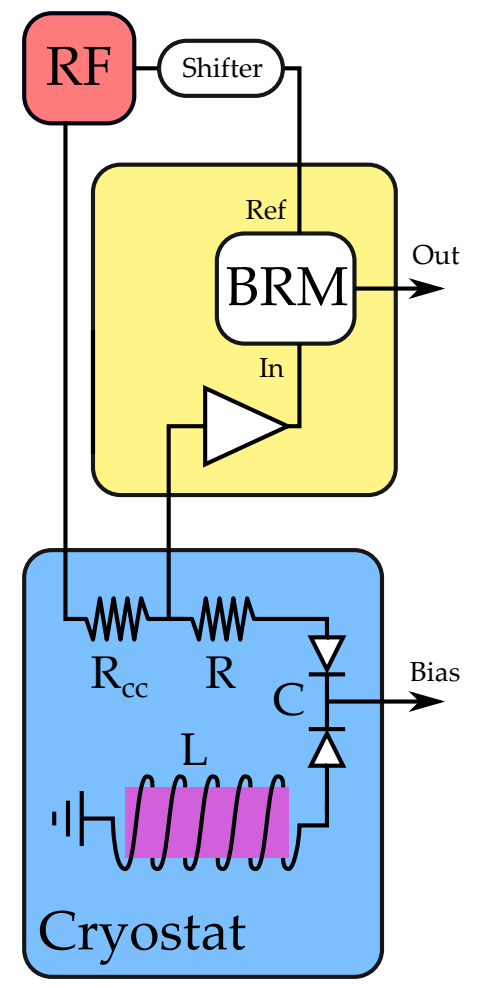

(b) “Cold" Q-meter NMR

Figure 2: Diagrams showing the traditional NMR layout, and the new, "cold" circuit NMR layout, which includes varactor-diode tuning capacitors and an electronic phase shifter. 


\subsection{Tests of Cold NMR at 5 T, $77 \mathrm{~K}$}

Our first focus was the creation of a convenient testbed to allow the investigation of NMR techniques without the added expense and effort needed to perform efficient DNP at $5 \mathrm{~T}$ and $1 \mathrm{~K}$. By performing NMR at $77 \mathrm{~K}$ rather than $1 \mathrm{~K}$, any thermal equilibrium polarization in a material would be roughly 50 times lower. However the resulting signal loss would be offset by the convenience of cooling with a simple, open bath of liquid nitrogen. Demonstrating the feasibility of NMR techniques at $77 \mathrm{~K}$ and in a $5 \mathrm{~T}$ warm bore magnet will show their robustness in very disadvantageous conditions.

Our $77 \mathrm{~K}$ test bed utilizes an insulated dewar that extends into the 4-inch warm bore of a $5 \mathrm{~T}$ solenoid magnet. A garolite insert held the material sample and NMR circuit in the center of the magnet, and could be retracted into the main volume of the dewar for access. For initial tests, $2 \mathrm{~cm}$-diameter-loop NMR coils were embedded in an epoxy sample to ensure strong magnetic coupling. By doping this epoxy with TEMPO [10], it was also possible to polarize the epoxy with microwaves. First, this coil was tested with a traditional Q-meter, on the end of a $\lambda / 2$ cable, resulting in a small, but manageable signal, $0.05 \mathrm{mV}$ in height above a noise floor of approximately $0.008 \mathrm{mV}$, seen in figure 3a. Next, a "cold" NMR board previously used in Hall B was tested, giving a $0.15 \mathrm{mV}$ signal over a $0.009 \mathrm{mV}$ noise floor. These two measurements of a thermal equilibrium signal in epoxy showed not only the feasibility of our tests at $5 \mathrm{~T}$ and $77 \mathrm{~K}$, but also demonstrated the roughly factor of 3 improvement of signal to noise offered by the cold circuit NMR technique.

To evaluate remote tuning, we produced prototype "cold" circuit NMR boards with GaAs, varactor-diode, tuning capacitors [11]. Using a tuning voltage between 0 and $14 \mathrm{~V}$ in a reverse bias on the varactors can produce as little as $0.2 \mathrm{pF}$ or as much as $20 \mathrm{pF}$ of capacitance per diode, depending on the model used. Prior to their use in these tests, we verified the operation of these diodes down to $3.2 \mathrm{~K}$ using a pulse-tube cryocooler setup. To allow remote tuning of the phase shift required to match signal and reference, we utilized a Mini-Circuits phase shifter [12], which can shift phase by up to $180^{\circ}$ and is controlled by a bias voltage of 0 to $15 \mathrm{~V}$. This phase shifter introduced negligible noise to our measurements.

Figure $3 \mathrm{~b}$ shows a signal from our new cold NMR setup, including the electronic phase shifter. This $5 \mathrm{~T}, 77 \mathrm{~K}$ thermal equilibrium measurement uses the same $2 \mathrm{~cm}$, epoxy embedded coil. The signal is approximately as strong as that obtain with the legacy Hall B cold board, $0.15 \mathrm{mV}$. The noise floor is lower here, $0.006 \mathrm{mV}$, but this is due to longer signal averaging. In practice the signal to noise between the new varactor-controlled board and the legacy, static capacitor board was similar. With this proof-of-concept obtained, we attempted to find a TE signal at room temperature and $5 \mathrm{~T}$. This was successful, resulting a signal roughly 4 times smaller, but still easily resolved from the noise. DNP at $77 \mathrm{~K}$ using microwaves was also explored, but the first investigations resulted in only moderate polarization enhancement of three or four times the thermal equilibrium value.

\subsection{Two Cell Tests at 5 T, $77 \mathrm{~K}$}

The $77 \mathrm{~K}$ testbed was then altered to allow NMR measurements of two cells simultaneously in a setup that would mimic the final target geometry. Figure 4a shows a rendering of the twocell test setup. The evacuated, stainless steel dewar has concentric garolite tubes that extend into 


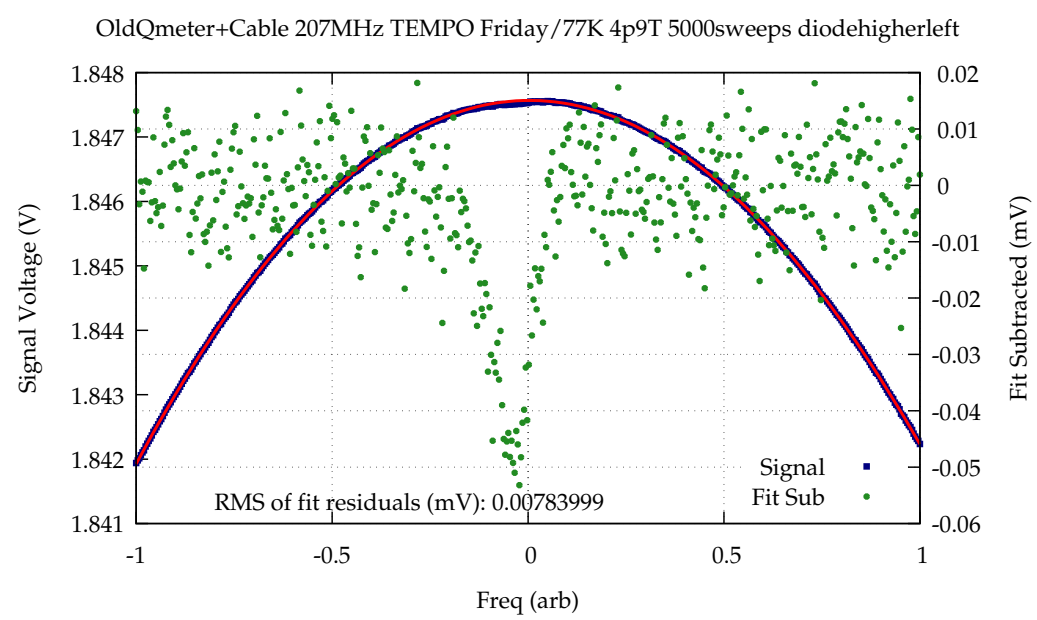

(a) TE signal using the traditional, resonant cable Q-meter measurement.

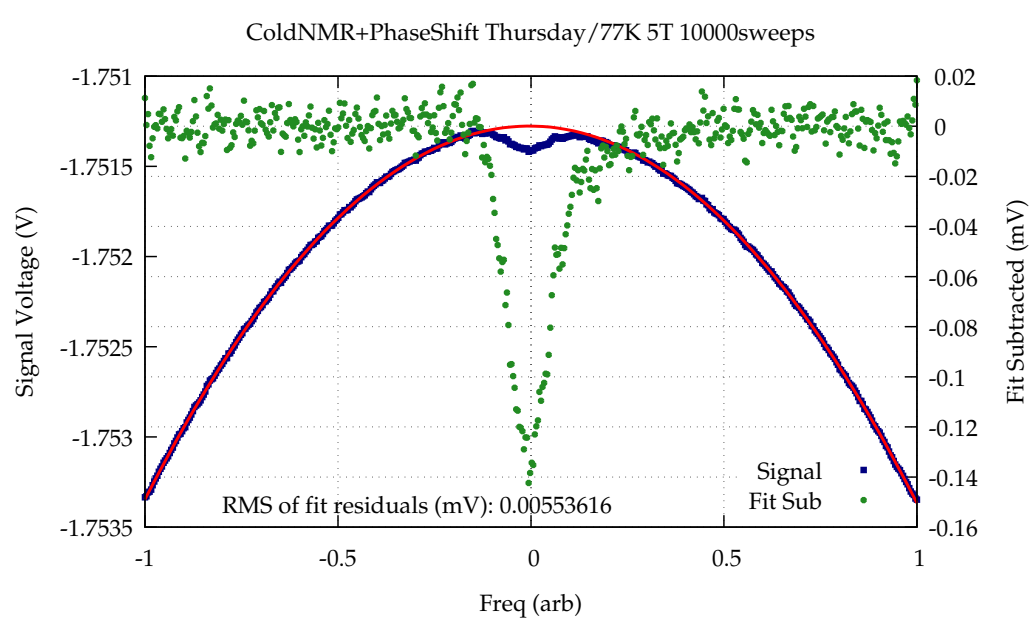

(b) TE signal from our new, varactor-based, "cold" NMR circuit.

Figure 3: Thermal equilibrium signals at $5 \mathrm{~T}$ and $77 \mathrm{~K}$ for traditional and cold circuit NMR boards. Again, the raw signal is in blue, with a background fit in red and the final, subtracted signal in green.

the warm bore and are capped with kapton windows, allowing microwaves to enter from the far side. Within these, an aluminum carrier holds 2 sets of test copper shimming coils, allowing the production of two distinct magnetic field regions. Within this are two test material samples (HDPE in this case) held in a PTFE insert ladder, which also carries the NMR coils. PTFE was chosen to avoid introduction of excess hydrogen material which would interfere with our proton NMR measurements.

For these tests, we mounted the NMR coils on the exterior of the PTFE mounting ladder, mimicking the external coils to be required in the final target. Figure $4 \mathrm{~b}$ shows the NMR tank board and one of two sets of copper coils laminated in PFA and mounted on either side of the PTFE ladder. Here a single set of coils is being used in parallel to observe both HDPE test samples, 


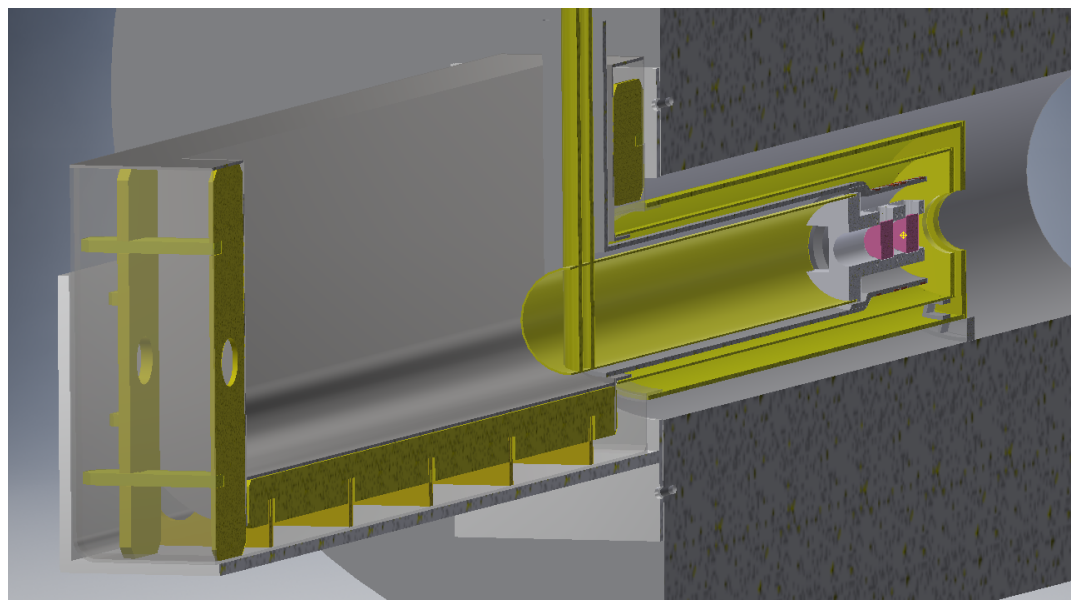

(a) A CAD rendering of the $77 \mathrm{~K}$ test bed. Starting at the center of the magnet and concentrically working out, the rendering shows two test samples (purple) held by the PTFE ladder, aluminum carrier for the 4 shim coils, two garolite (yellow) tubes of the evacuated dewar, and the magnet itself. The outer shell of the dewar is transparent to show the garolite support ribbing.

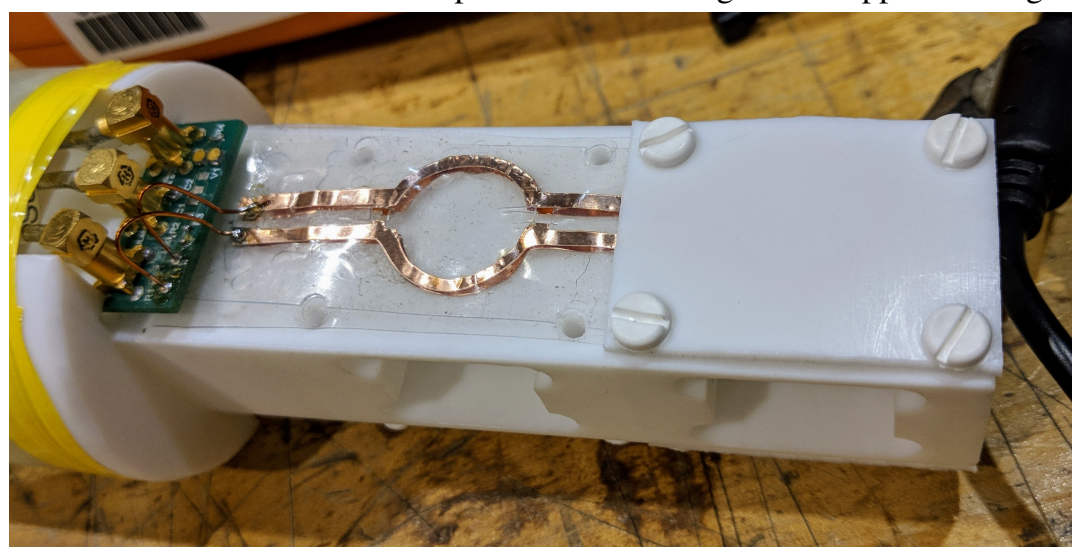

(b) Photo of PTFE mounting ladder showing the NMR tank circuit board at left soldered to a two-layer copper coil laminated in PFA, which is held in place by one of the two PTFE covers. A second coil set is mounted to the opposite side of the ladder (although not yet connected to the board).

Figure 4: Experimental layout for the $5 \mathrm{~T}, 77 \mathrm{~K}$ tests, using two sample cells and cold circuit NMR board.

rather than using separate coils for each cell which would require separate NMR boards and Qmeters. Coils large enough to cover both cells have much higher inductance than a simple $2 \mathrm{~cm}$ loop, complicating the tuning at $213 \mathrm{MHz}$. We utilized 4 coils in parallel to reduce the inductance to within the usual range of our capacitors-around $40 \mathrm{nH}$ and $12 \mathrm{pF}$ for $213 \mathrm{MHz}$. If the inductance is too high and tuning capacitance too low, the effect of parallel stray capacitance to ground becomes non-trivial.

The shim coils were wound around an aluminum mandrel, and their resulting field was simulated. The simulation included a minimization routine allowing determination of the optimal 
current required in each shim coil to produce a desired field on top of the $5 \mathrm{~T}$ solenoid field. For these tests, the field in the region of each cell was moved by 50 gauss in either direction.

Figure 5 shows NMR thermal equilibrium signals at $5 \mathrm{~T}$ and $77 \mathrm{~K}$ using external coils covering both cells. In figure 5a, the shims are not energized, so that the NMR signal from the protons in the two HDPE samples stack on top of one another, resulting in a curve similar to that in figure 3. The noise floor is markedly worse due to difficulties in maintaining a steady tune with the large NMR coils. When the shims were energized to \pm 50 gauss, the change in field resulted in a shift of the Larmor frequencies in either sample. This separates the portion of the signal from each HDPE sample, creating two peaks at above and below the original Larmor frequency, seen in figure 5b. Although we again experienced difficulty maintaining a steady tune, we were able to resolve both peaks, each roughly half the size of the joint signal with the shims off.

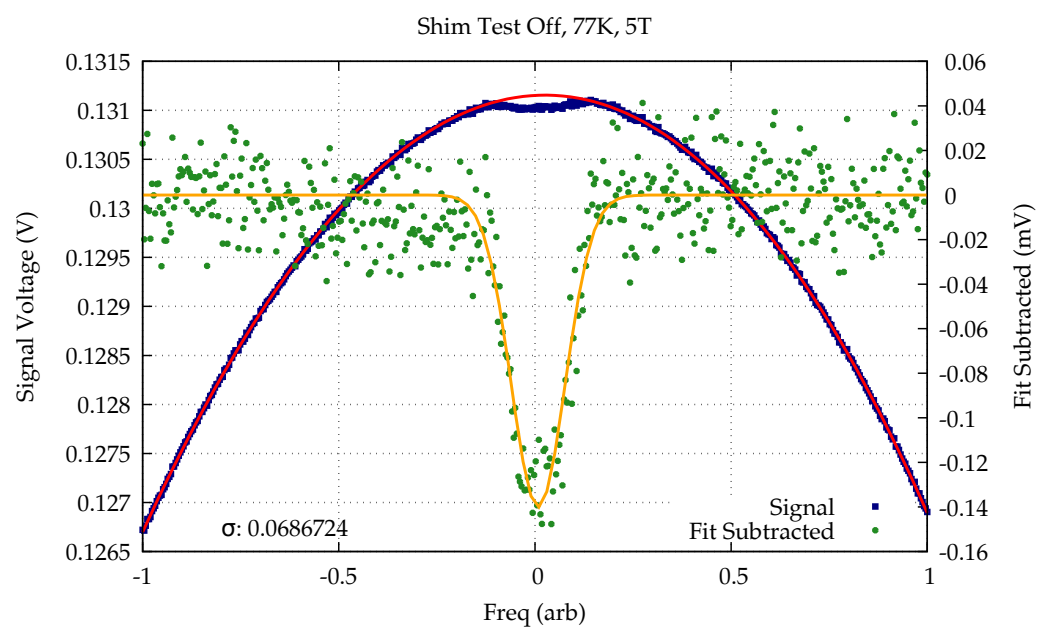

(a) TE signal with shims unenergized.

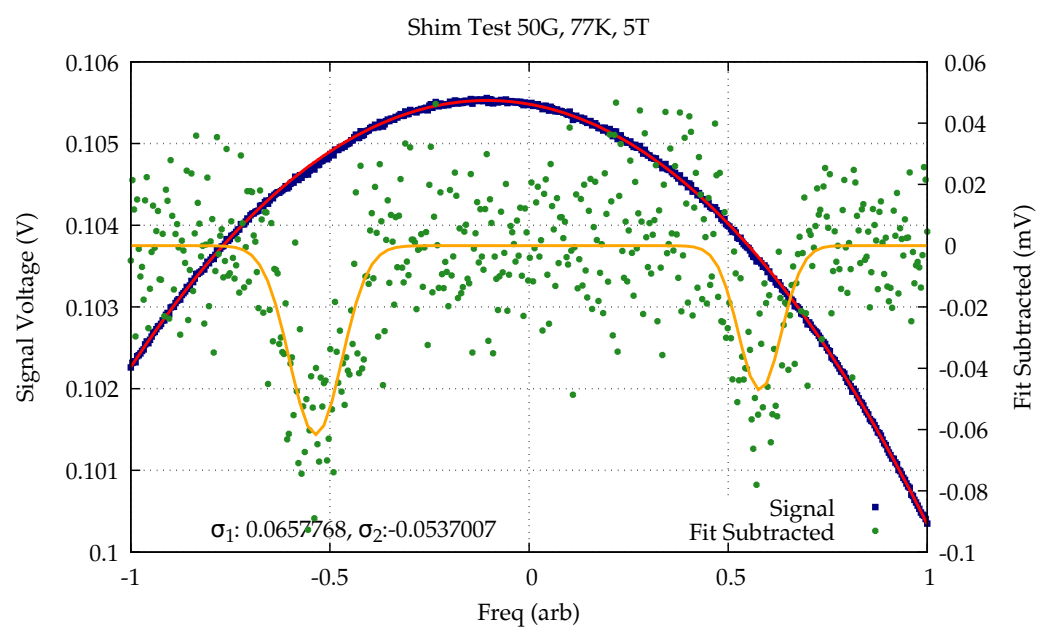

(b) TE signal with shims energized, producing \pm 50 gauss and separating the contributions from the two different samples.

Figure 5: TE signals using our cold circuit viewing large coils covering both cells. The subtracted signals in green are fit with Gaussians in orange to guide the eye. 


\section{Conclusions and Outlook}

As the first experiments utilizing the new CLAS12 polarized target approach in the next several years, the required incremental improvements in NMR polarization measurements are on track. We have demonstrated the efficacy of tests of our Q-meter NMR systems at $77 \mathrm{~K}$, and even resolved a thermal equilibrium signal at room temperature. Using this testbed, we have shown the feasibility of remotely tuned, external NMR coil measurements in a two cell setup. The tests of magnetic field shimming support the possibility of running two cells at opposing polarizations with a single microwave frequency. We intend to continue with tests of DNP at $77 \mathrm{~K}$ for opposing cells in the coming months.

Looking forward, we are exploring the renovation and replacement of the aging Liverpool Q-meters. As they are no longer sold, parts which fail can be difficult to replace. With the collaboration of the JLab Fast Electronics group, we have begun producing a new Q-meter, following the original closely but using modern components (much like the Bochum effort [13]). In an attempt to reduce noise, we are also investigating the introduction of a digitizer inside the Q-meter chassis, as well as the possibility of including an amplifier on the cold NMR circuit. For the eventual replacement of the Liverpool Q-meter, we are exploring all-digital solutions, either leveraging down mixing or very fast ADC digitization.

\section{Acknowledgments}

Many thanks to collaborator V. Lagerquist of Old Dominion University for the winding and simulation of shim coils. This material is based on work supported by the U.S. Department of Energy, Office of Science, Office of Nuclear Physics under contract DE-AC05-06OR23177.

\section{References}

[1] D. G. Crabb and W. Meyer, Solid polarized targets for nuclear and particle physics experiments, Annual Review of Nuclear and Particle Science 47 (1997) 67.

[2] G. Court, D. Gifford, P. Harrison, W. Heyes and M. Houlden, A high precision q-meter for the measurement of proton polarization in polarised targets, Nuclear Instruments and Methods in Physics Research Section A 324 (1993) 433.

[3] C. Keith, A dynamically polarized target for CLAS12, in These Proceedings, 2017.

[4] D. Adams et al., The polarized double cell target of the smc, Nuclear Instruments and Methods in Physics Research Section A 437 (1999) 23.

[5] K. Kondo et al., Polarization measurement in the compass polarized target, Nuclear Instruments and Methods in Physics Research Section A 526 (2004) 70.

[6] P. McKee, Observations of radiation damage and recovery in ammonia targets, Nuclear Instruments and Methods in Physics Research Section A 526 (2004) 60 .

[7] J. Maxwell et al., Design and performance of the spin asymmetries of the nucleon experiment, Nuclear Instruments and Methods in Physics Research Section 885 (2018) 145. 
[8] G. Court, M. Houlden, S. Bultmann, D. Crabb, D. Day, Y. Prok et al., High precision measurement of the polarization in solid state polarized targets using $\mathrm{nmr}$, Nuclear Instruments and Methods in Physics Research Section A 527 (2004) 253.

[9] E. J. Veenendaal, R. Hulstman and H. B. Brom, A frequency-modulated q-meter for very low-temperature nmr experiments, Journal of Physics E: Scientific Instruments 16 (1983) 649.

[10] Y. Noda, T. Kumada, D. Yamaguchi and S. Shamoto, Thermosetting polymer for dynamic nuclear polarization: Solidification of an epoxy resin mixture including tempo, Nuclear Instruments and Methods in Physics Research Section A 776 (2015) 8.

[11] MACOM, "Macom MA46 series GaAs tuning varactors."

[12] MiniCircuits, "Surface mount phase shifter SPHS-251+."

[13] J. Herick, Development of a new Q-meter module, PoS PSTP2015 (2016) 011. 